\title{
Proceso de inventariado y catalogación del pasado arqueológico en la isla de Tenerife
}

\author{
The Process of Inventory and Cataloging of the Archaeological Past in \\ Tenerife
}

\author{
Gisela de la Guardia Montesdeoca \\ Universidad de La Laguna \\ https:/ / orcid.org/0000-0002-6815-9013 \\ giselaguarmon@gmail.com
}

Recibido: 19/07/2021; Revisado: 16/10/2021; Aceptado: 09/12/2021

\begin{abstract}
Resumen
Este artículo supone un acercamiento a la evolución del proceso de inventariado efectuado en la isla de Tenerife hasta la actualidad. El objetivo en torno al cual gira este estudio se refiere a la actualización de los datos recogidos por las principales líneas de investigación, así como su contextualización. Mediante el uso de una metodología eminentemente cualitativa en base al análisis de la bibliografía científica y de las fuentes custodiadas por la Dirección General de Patrimonio Cultural se pueden inferir una serie de conclusiones por la cual en la isla de Tenerife ha primado el trabajo de conocimiento de su pasado en detrimento de otras áreas de la gestión, como la difusión.
\end{abstract}

Palabras clave: Gestión, catalogación, inventariado, patrimonio arqueológico.

\begin{abstract}
This article looks at the evolution of the inventory process implemented on the island of Tenerife. The article's main objective is the contextualization of the latest data collected by the main lines of research. Through the use of an eminently qualitative methodology, based on the analysis of scientific bibliographies and sources deriving from the General Directorate of Cultural Heritage, a series of conclusions can be drawn, suggesting that on the island of Tenerife work to study its past has taken precedence over other areas of management, to the detriment of processes such as dissemination..
\end{abstract}

Keywords: Management, Cataloging, Inventory, Archaeological Heritage. 


\section{INTRODUCCIÓN}

Este trabajo analiza la evolución en el proceso de inventariado y catalogación en la isla de Tenerife, desde los años ochenta hasta la actualidad. Las investigaciones en torno a la construcción del conocimiento del pasado isleño desde la perspectiva de las áreas aludidas, encuentra una referencia de excepción en la tesis doctoral de Elena $\mathrm{M}^{\mathrm{a}}$ Pérez (2014) que realiza un exhaustivo análisis de la documentación custodiada en el archivo de la Dirección General de Patrimonio Cultural. Adicionalmente, la bibliografía tinerfeña se complementa con la producción científica cuya autoría se atribuye a investigadores como NAVARRO (1997), TEJERA (1992) o CABRERA $(2007,2019)$.

El objetivo principal de este estudio se referirá, por consiguiente, en determinar los fines que mueven las intervenciones arqueológicas efectuadas en la isla de Tenerife desde la década de los años ochenta hasta la actualidad. Todo ello para definir la representación de cada una de las áreas que componen la gestión patrimonial como son conocer, planificar, controlar y difundir (QUEROL, 2010: 52-56).

El texto que aquí se presenta se ha estructurado en función de una serie de axiomas, partiendo de lo general (antecedentes, estado de la cuestión, objetivos) a lo específico. Este último campo se ha estructurado de la misma manera, partiendo de los rasgos generales que caracterizan las intervenciones llevadas a cabo en las islas y en el caso particular tinerfeño. A partir de este análisis se procederá a tipificar las peculiaridades que adquiere el proceso de inventariado y catalogación en la isla para concluir con una concisa exposición de conclusiones.

\section{ANTECEDENTES}

Las vías para emprender el proceso de generación de conocimiento en las islas confluyen en la figura de la intervención, entendida como una actuación con mayor o menor impacto en los bienes arqueológicos, tanto muebles como inmuebles. En virtud de ello, las distintas intervenciones quedan categorizadas según el Decreto 262/2003 de 23 de septiembre, por el que se aprueba el Reglamento sobre intervenciones arqueológicas en la Comunidad Autónoma de Canarias, quedando establecidas en las categorías que siguen:

- Excavaciones.

- Sondeos.

- Prospecciones.

- Reproducción de manifestaciones rupestres.

- Estudio de materiales en museos.

Tras las actualizaciones pertinentes contenidas en la Ley 11/2019 de Patrimonio Cultural de Canarias, se revisan estas tipologías, resultando el hecho de que las actividades arqueológicas se clasifican en función del uso de la metodología arqueológica, al margen de su periodo histórico, tanto terrestres como en medio acuáticos (art. 90). Así, quedarán dictaminadas en las siguientes:

- Excavación arqueológica: tanto en superficie como bajo el mar, con la finalidad de descubrir, documentar o investigar restos arqueológicos.

- Sondeo: remoción de terreno con el objetivo de comprobar la existencia 
de restos muebles e inmuebles.

- Prospección: exploración superficial (superficial o subacuática), para la detección de restos.

- Reproducción de manifestaciones rupestres de interés histórico.

- Consolidación y restauración: nuevo tipo de intervención incorporada para favorecer tanto su conservación como su puesta en valor, es decir, poniendo el punto de mira en la difusión.

- Control arqueológico: de nuevo otra de las novedades en cuanto a actividades, enfocada a la supervisión por parte de personal técnico cualificado de obras que puedan afectar a bienes arqueológicos.

- Análisis estratigráfico de estructuras arquitectónicas: aplicar técnicas propias de la metodología arqueológica para documentar e investigar secuencias históricas en edificaciones (lectura de paramentos o aplicación de la arqueología de la arquitectura).

- Estudio de materiales arqueológicos: elemento novedoso que carece de explicación añadida, más que su enunciación.

Podemos agrupar las intervenciones, por tanto, sobre la base del impacto que generan en los bienes en cuestión, en el que la excavación constituye la acción que mayor incidencia tiene sobre el patrimonio. De ahí que se alcen voces defendiendo o priorizando intervenciones de menor impacto como son los sondeos o la prospección, dentro de un movimiento de mayor envergadura denominado arqueología preventiva (QUEROL, 1995).

\subsection{Estado de la cuestión}

Para elaborar este epígrafe, nos vamos a remitir a los datos extraídos de la tesis doctoral de Elena M M $^{\mathrm{a}}$ Pérez (2014) en la cual precisa las áreas de gestión del patrimonio arqueológico en las islas, a través de un exhaustivo análisis de los expedientes de autorizaciones custodiados por la Dirección General de Patrimonio Cultural. Así pues, su investigación ha permitido establecer un panorama genérico de la situación desde la creación de la administración autonómica en 1984 hasta comienzos del año 2011.

\subsection{Metodología y fuentes}

Para ejecutar este trabajo, se ha utilizado una propuesta metodológica sustentada en la investigación cualitativa, definida como el proceso por el cual se entiende, describe y, en ocasiones, se explican fenómenos sociales por medio de distintos procedimientos, entre los que se incluye el análisis de fuentes diversas (BANKS, 2010).

El proceso de análisis se basará, de esta manera, en los siguientes referentes:

- Investigación bibliográfica afín, dentro del contexto estatal e insular

- Archivo de la Dirección General de Patrimonio Cultural del Gobierno de Canarias Hemeroteca de la Biblioteca de la Universidad de La Laguna. 


\subsection{Breve repaso normativo}

Las recomendaciones internacionales posteriores a la Segunda Guerra Mundial han insistido en la necesidad de autorizar y "vigilar» (Nueva Delhi, 1956) las intervenciones realizadas en el patrimonio arqueológico. En lo sucesivo, a nivel internacional declararán la necesidad de asegurar que cualquier acción que suponga una afección al patrimonio arqueológico deba ser realizada por personal cualificado (La Valetta, 1992), empero, sin quedar estipulado qué conlleva esa cualificación (Pérez, 2014: 143), teniendo en consideración que, hasta tiempos recientes, España carecía de titulación específica en el área de arqueología.

Ya en territorio nacional, desde el siglo XIX, España es consciente de la necesidad de regular las intervenciones arqueológicas, produciéndose un punto de inflexión a partir de la promulgación de la Real Cédula de 6 de julio de 1803, que disponía «el modo de recoger y conservar los monumentos antiguos descubiertos o que se descubran en el Reino». No obstante, no sería hasta comienzos del siglo xx cuando se materializa un impulso decidido por parte de las autoridades hacia la protección de los restos del pasado, a partir de un hito de especial relevancia como fue la publicación oficial de la Ley de 7 de julio sobre excavaciones arqueológicas de 1911. Hasta entonces, el conjunto de la normativa no aspiraba a ser un cuerpo legislativo bien estructurado, sino más bien, un conglomerado de normas y preceptos legales aislados y sin coordinación (BUGELLA, 2017: 321). Así, esta ley introduce una serie de elementos originales como son la definición de «excavación» («remoción deliberada y metódica de terrenos respecto a los cuales existan indicios de yacimientos arqueológicos, ya sean restos de construcciones, o antigüedades»), el requerimiento de realizar un inventario estatal de «las ruinas monumentales», la necesidad de solicitar autorización para realizar excavaciones y una nada deleznable explicación sobre la potestad sobre bienes descubiertos.

\section{RESULTADOS}

Ante todo, el caso canario muestra sus particularidades en el hecho isleño y en el modelo de gestión patrimonial. Partiendo del análisis cuantitativo y cualitativo de las intervenciones efectuadas en el rango de años señalados, se evidencia que la gran mayoría de las autorizaciones a nivel autonómico se corresponden con prospecciones, seguidas de excavaciones arqueológicas, trabajos de estudio y documentación, y, por último, actividades de restauración y/o conservación (PÉREZ, 2014: 190).

Tras el cribado inicial de los 917 expedientes analizados por PéRez (2014: 164), el resultado definitivo es un total de 862 autorizaciones de intervención para ese periodo de años, correspondiendo 260 a la isla de Tenerife (Pérez, 2014: 182).

Las premisas de análisis que se extraen a partir del estudio de Pérez pueden agruparse en los siguientes postulados:

- La provincia de Las Palmas de Gran Canaria concentra el mayor número de intervenciones autorizadas, en relación con la provincia vecina. Este hecho puede explicarse por la confluencia de una serie de factores como ser la sede de la Dirección General de Patrimonio Cultural y Cooperación, de la Universidad de las Palmas de Gran Canaria y de la gran mayoría de empresas privadas de 
arqueología (PÉREZ, 2014, 181-182). Por otra parte, la preponderancia de la isla vecina en relación con las intervenciones arqueológicas fue ya reseñada a mediados de los años noventa por José Juan JiménEz (1995: 30).

- Existencia de una completa capitalización de las intervenciones, es decir, un mayor protagonismo de las dos islas capitalinas en relación con las restantes (Pérez, 2014: 180). De nuevo tenemos que remitirnos a la posible hipótesis de situación de las principales instituciones políticas, universitarias, así como empresas privadas del sector.

- Aumento más acusado de las intervenciones arqueológicas en la provincia de Las Palmas a partir de los años noventa (Pérez, 2014: 183).

- Más de un tercio de las intervenciones llevadas a cabo en las islas, han sido efectuadas por profesionales libres, en detrimento de la iniciativa pública, las empresas, los museos arqueológicos y otras instituciones (PÉrEZ, 2014: 187).

Como indica Pérez (2014: 187), no pudieron ser identificadas la totalidad de las causas que motivaron su desarrollo, aunque sí se puede señalar el hecho de que el motor que impulsó la mayoría fue de índole urbana o constructiva, seguido de informes de evaluación de impacto ambiental y la elaboración de inventarios de yacimientos arqueológicos. En menor medida, el resto de los motivos contemplados han sido encargos puntuales o limpieza de entornos patrimoniales, siendo testimonial las actividades destinadas a la difusión (PÉrEZ, 2014: 195).

De esta suerte, la excavación de urgencia se convierte en un recurso de lo más usual en las intervenciones arqueológicas, con un predominio de los intereses empresariales que da lugar, en última instancia, a que en Canarias obtengamos un conocimiento fragmentario, al haber dejado de lado el desarrollo de grandes proyectos de investigación, a favor de acciones puntuales, haciendo patente la falta de coordinación no sólo institucional, sino de las mismas acciones en sí, en un contexto que reclama y reivindica la cooperación ante la ineficacia institucional.

En cuanto a la escasa representación de actividades de difusión, la autora puntualiza el que, si bien su número es anecdótico, no puede pasarse por alto el que muchas propuestas de revalorización no conllevan en todos los casos, la solicitud de autorización de intervención por lo que son datos que no formaban parte de su fuente de consulta (PÉREZ, 2014: 196).

A propósito de la evolución del número de autorizaciones en la isla de Tenerife, es preciso señalar que la transferencia de competencias en materia patrimonial y cultural al gobierno autonómico procuró un mayor suministro de fondos para investigación, permitiendo que las campañas de estudio se integraran en proyectos de mayor amplitud evaluados por una comisión asesora, después ponencia técnica (NAVARRO, 1997). Este hecho determinaría la reducción de las intervenciones, pero, por otra parte, mejorando su calidad (NAVARRO, 1997).

En el año 1987 se abre una vía de financiación autonómica en vistas a la realización de proyectos de investigación, siendo este el motor de la mayoría de las intervenciones efectuadas hasta finales del año 1991. Este año clave supone, no sólo la suspensión de una financiación fundamental sino, también, el trasvase definitivo de competencias en materia patrimonial a la recién creada Dirección General de Patrimonio Histórico (PÉREZ, 2014, 204-205). La finalización de esta ayuda, vital para la gestión de proyectos de investigación, provocaría, en última instancia, la pérdida hasta hoy del protagonismo de la universidad en la investigación arqueológica (JIMÉnez y MEDEROS, 2001) y la desaparición de proyectos de investigación consolidados (Pérez, 2014, p.210). 
Entre los años 1993 y el 2002 se abre una nueva vía de financiación mediante subvenciones destinadas al estudio en materia de patrimonio histórico (Decreto 31/1993, de 5 de marzo, por el que se establece el régimen general de ayudas y subvenciones de la Administración Autonómica de Canarias y la Orden de 5 de octubre de 1993, por la que se convoca y regula la concesión, por el procedimiento de concurso, de subvenciones para el estudio y documentación del Patrimonio Histórico de Canarias), e incluiría la realización de excavaciones, prospecciones y sondeos en diferentes entornos patrimoniales, la elaboración de actividades de inventariado y catalogación, investigación, y diversas actividades de difusión y divulgación del conocimiento patrimonial, con la gran novedad de introducir, por vez primera en convocatorias de esta naturaleza, la puesta en uso del Patrimonio Histórico con fines turísticos (PÉREZ, 2014: 207). Este nuevo impulso a la difusión patrimonial daría lugar a hitos preponderantes en la historia de la disciplina como son la apertura al público de los entornos de la Cueva Pintada de Gáldar en Gran Canaria, los grabados de La Zarza y La Zarcita en La Palma o los de El Julan en El Hierro (PÉREZ, 2014: 208).

Las intervenciones efectuadas a partir de la promulgación de la Ley 4/1999 de Patrimonio Histórico de Canarias serán promovidas, en mayor medida, desde los cabildos insulares, empresas privadas y una pequeña representación de los ayuntamientos, inaugurando un nuevo periodo marcado por lo que algunos autores tildan como profunda crisis que afectará en última instancia a la investigación (NAVARRO et al., 2005).

En el caso de la isla de Tenerife, Pérez establece un modelo evolutivo siguiendo las desavenencias producidas en materia política con cada cambio de legislatura. El resultado último, tras el trasvase de competencias, ha sido un entorno profundamente atomizado, en el que los Cabildos ejecutan sus planes de acción de manera paralela y al margen del Gobierno de Canarias (PÉREZ, 2014, 197). Todo ello coincide, igualmente, con la pérdida a finales de los noventa del otrora protagonismo de las universidades canarias, viendo marginadas sus posibilidades de financiación y actuación, en un panorama copado por los gobiernos insulares.

\section{INTERVENCIONES ARQUEOLÓGICAS RECIENTES (2011-2017)}

La consulta del archivo de la Dirección General de Patrimonio Cultural del Gobierno de Canarias ha permitido mantener la vigencia de la práctica totalidad de los postulados establecidos por Pérez (2014) en su trabajo doctoral. Conforme a los datos extraídos, contabilizamos para el periodo del 2011 hasta el 2017, un total de 62 intervenciones realizadas, sin contar las desestimadas (Gráfico 1). 


\section{GRÁFICO 1}

Evolución del número de intervenciones en la isla de Tenerife entre el año 2011 y el 2017

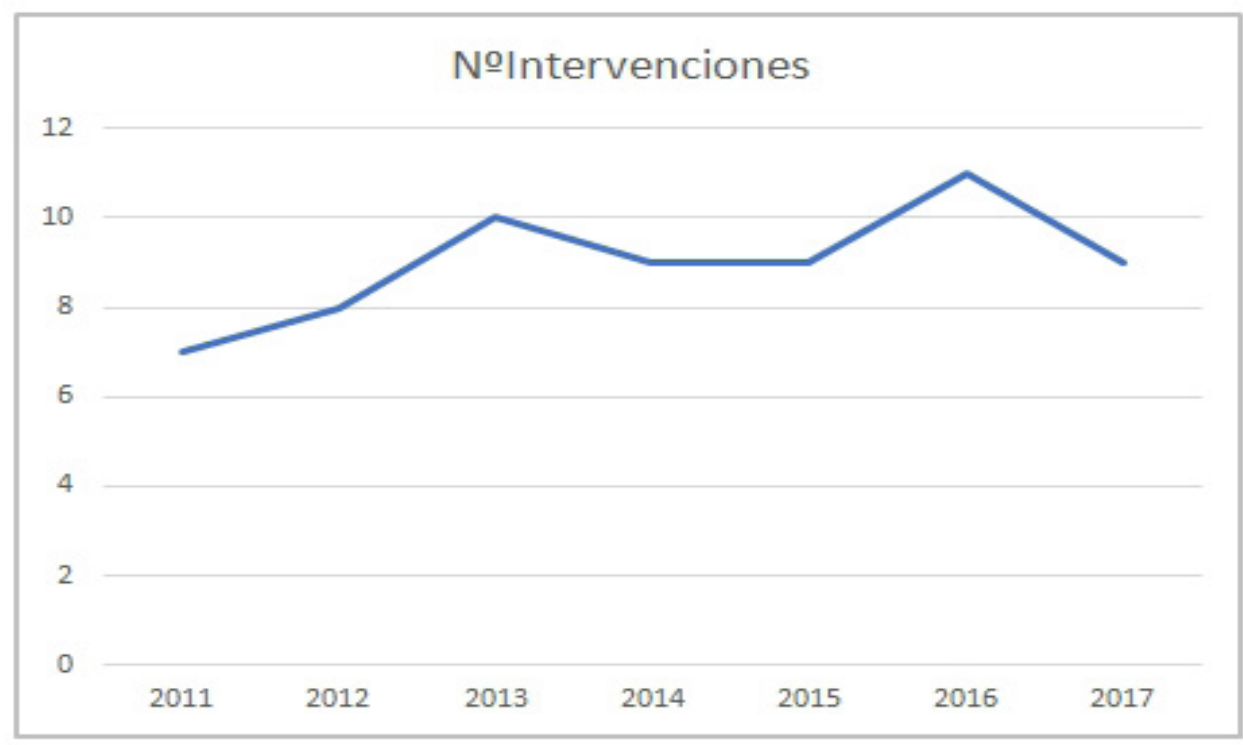

Fuente: Elaboración propia.

En vistas a los datos obtenidos, observamos un ligero aumento en el rango de años entre el 2013 y 2016, en las intervenciones efectuadas en la isla de Tenerife.

En el transcurso del año 2013, del total de intervenciones realizadas, el mayor porcentaje se engloba en el parámetro que hemos denominado intervenciones vinculadas a procesos constructivos, frente a aquellas intervenciones vinculadas a investigación. Dentro de los procesos constructivos implicados, se encuentran reformas de líneas de media tensión, polígonos industriales o parques eólicos. Incluida dentro de la designación de intervenciones con fines investigadores destacan estudios en el área del Parque Nacional del Teide. En ese mismo año, agrupado en el criterio otros destacamos la exhumación y estudio bioantropológico de los restos de Amaro Pargo en la Iglesia de Santo Domingo en La Laguna.

En el año 2016, el aumento de intervenciones se concentra de nuevo en acciones vinculadas a procesos urbanísticos en el entorno del Parque Nacional del Teide, dentro del proyecto de rehabilitación del centro de visitantes Cañada Blanca, así como el trazado de nuevos senderos. Igualmente, se efectuaron prospecciones a raíz de otros proyectos como pistas agrícolas o depuración de aguas residuales. Englobado en las acciones con fines investigadores destacamos las intervenciones de documentación y reproducción de manifestaciones rupestres y prospección de la isla de Tenerife. 
Proceso de inventario y catalogación del pasado arqueológico en la isla de Tenerife

GRÁFICO 2

Tipología de las intervenciones realizadas

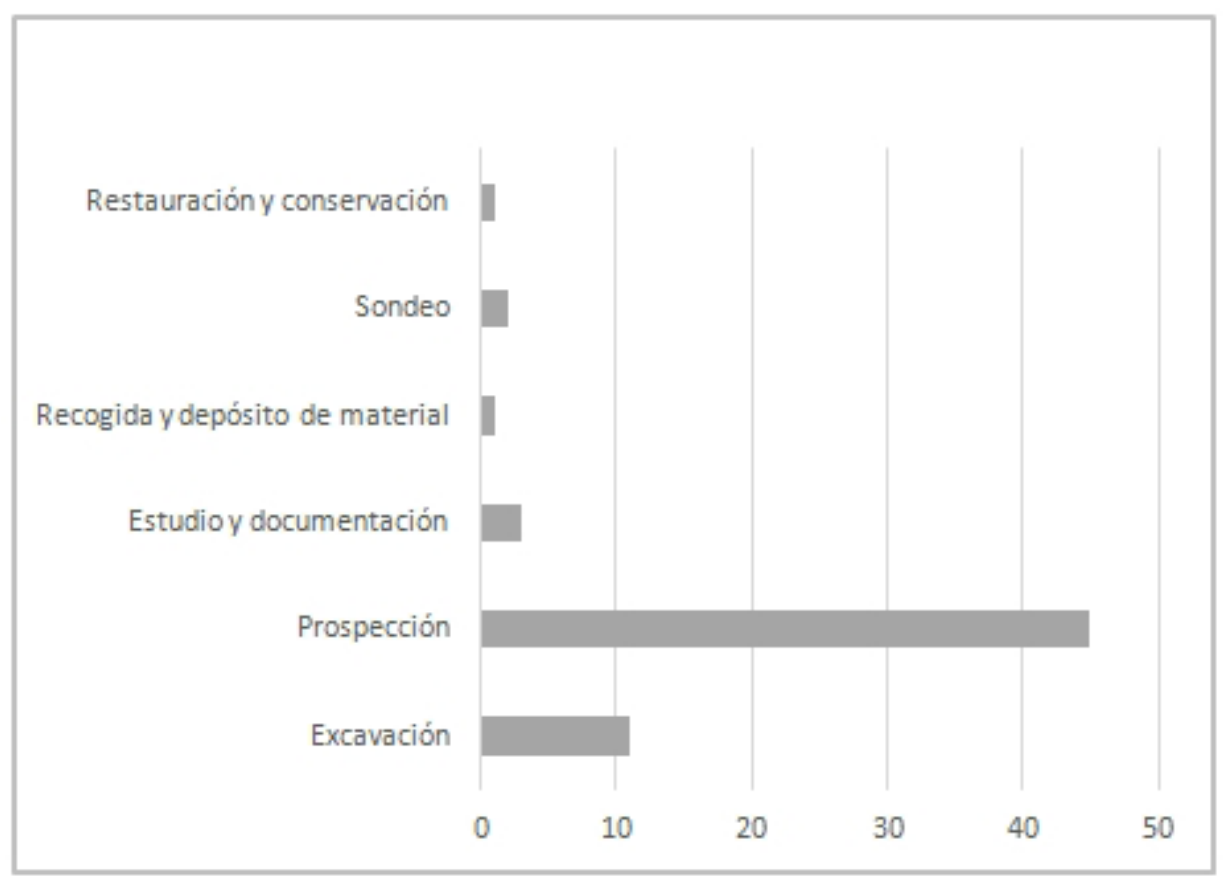

Fuente: Elaboración propia.

Las conclusiones que podemos extraer del análisis de las intervenciones en estos últimos años se pueden enunciar en las siguientes proposiciones (Gráfico 2):

- El grueso de las actividades se corresponde con prospecciones, seguidas de la excavación. Las prospecciones, en su mayoría, respondería a trabajos enmarcados dentro de los proyectos de evaluación del impacto ambiental, y una pequeña muestra a la elaboración de inventarios $(20 \%)$, suponiendo una ligera variación de la dinámica previa, aunque dado el tamaño del análisis no podemos extraer datos concluyentes.

- Se han efectuado dos intervenciones en entornos no aborígenes. Una vinculada a la recuperación de la memoria histórica y otra dentro de la arqueología histórica, con la acción realizada en la Plaza de los Remedios en el entorno de la Catedral de La Laguna.

- Realización de campañas planificadas vinculadas a proyectos de investigación, como es el caso de la llevada a cabo en las Cañadas del Teide por el equipo de la profesora de la Universidad de La Laguna, Matilde Arnay y la empresa PRORED.

- Las labores de estudio y documentación se refieren principalmente a actuaciones en localizaciones de grabados rupestres y a entornos que engloban un conjunto de bienes patrimoniales de distinta naturaleza.

- La única muestra de restauración y conservación quedaría englobada 
dentro de la arqueología histórica, entrando en conflicto con los planteamientos instaurados por la Ley 11/2019. En la misma, queda relegado el patrimonio arqueológico protegido a la esfera de lo aborigen, suponiendo un claro menoscabo a la necesidad de proteger el precario patrimonio isleño.

- Ausencia total de intervenciones destinadas a la preservación y mantenimiento de bienes arqueológicos, como el cerramiento y vallado de yacimientos o labores de restauración y conservación.

\section{CATALOGACIÓN DEL PATRIMONIO ARQUEOLÓGICO EN LA ISLA DE TENERIFE}

En el caso de la isla de Tenerife el proceso de inventariado ha adquirido un protagonismo inusitado, al contar con el indudable impulso recibido por parte del cabildo insular, que ha focalizado su actuación en esta área de trabajo en detrimento del resto de vertientes de la gestión.

El paso previo a la ejecución de un programa sólido de protección del pasado arqueológico pasa por la elaboración de un inventario exhaustivo que determine qué bienes son susceptibles de ser protegidos, siendo esta una función indispensable en la arqueología preventiva (QUEROL, 1992: 27) y una necesidad inexcusable en la gestión del patrimonio arqueológico (RAMOs et al., 1998; RODRÍGUEZ, 2004).

Desde el punto de vista conceptual, la literatura científica hace alusiones al proceso de catalogación e inventariado, casi utilizadas de manera indistinta, por lo que su posible diferencia residiría en su nivel de profundidad. Así, tradicionalmente se ha entendido el inventario como una simple mención, prácticamente un sencillo listado de bienes, mientras el catálogo tendría un nivel de mayor profundización en el conocimiento del bien.

A medida que se asienta el corpus terminológico en torno a la gestión patrimonial, la catalogación adquiere mayor amplitud conceptual, unido a la expansión de disquisiciones urbanísticas, dando lugar a su equiparación con los bienes inmuebles, quedando el inventario, por tanto, limitado a los bienes muebles. Ambos conceptos incluyen dos vertientes, la que incumbe a su definición en sí -conocimiento, investigación y difusión de una serie de elementos- y la relativa a su lado administrativo, puesto que, al quedar englobado en alguno de los listados citados, pasa a detentar un nuevo orden administrativo en el conjunto de bienes patrimoniales. En este sentido, Querol los distingue mediante la utilización de minúsculas y mayúsculas, hablando, por tanto, de inventarios de Patrimonio Cultural para los primeros y Bienes culturales Inventariados para los segundos (Querol, 2010: 64-67).

\section{PRIMEROS INVENTARIOS}

Las primeras cartas arqueológicas en España se remontan al primer cuarto del siglo XIX, con un carácter netamente estatal. No será hasta la transferencia de competencias de finales del siglo xx cuando comienza a contextualizarse en áreas 
más concretas el inventariado (MARTín et al., 1995), produciéndose en los ochenta el verdadero interés por parte de las autoridades para la confección de inventarios pormenorizados de los bienes arqueológicos.

En la realidad tinerfeña, la labor de inventariado contó con el impulso inicial de los primeros arqueólogos que dieron forma a la disciplina con Luis Diego Cuscoy a la cabeza que, en su faceta de arqueólogo, se lanzó a recorrer los parajes de la isla efectuando una sucinta catalogación al amparo de su hipótesis primordial en torno a la territorialidad de la sociedad aborigen.

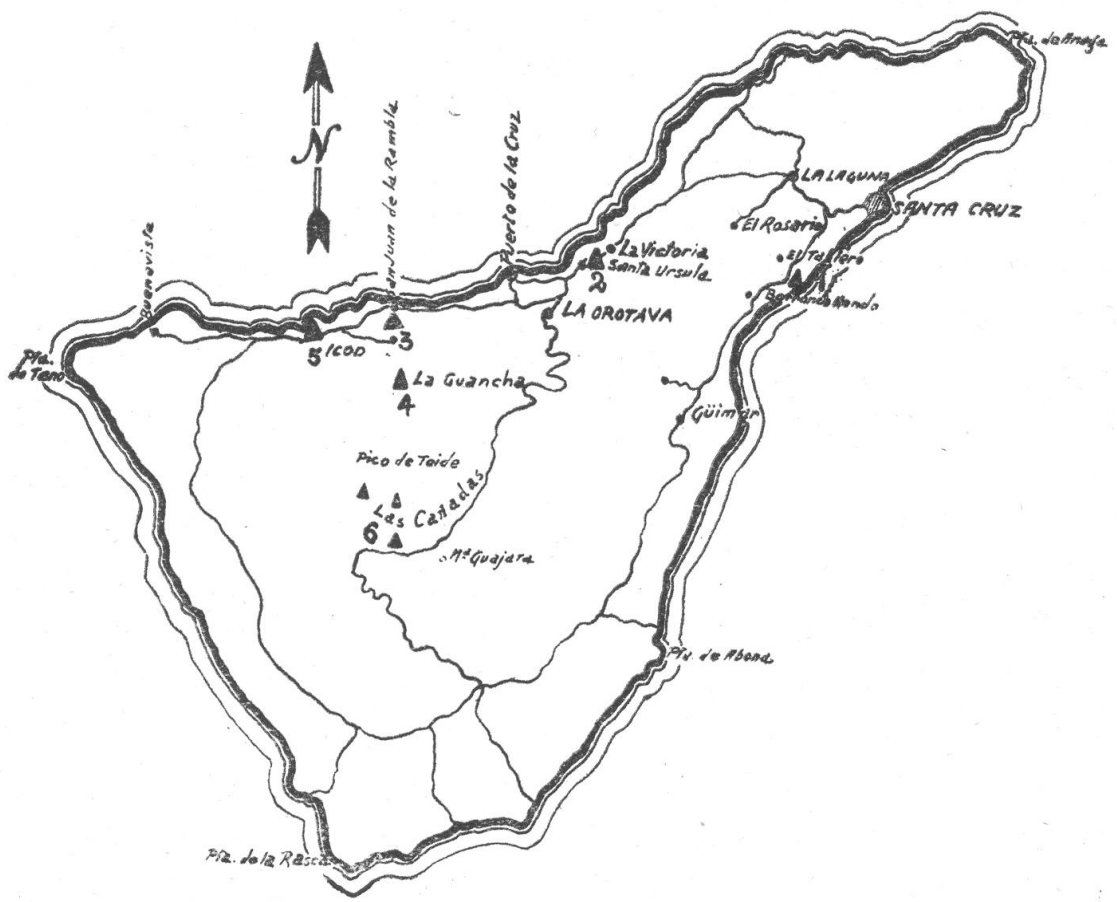

Figura 1. Localización de yacimientos sobre el mapa de la Isla de Tenerife. Fuente: DiEgo Cuscoy (1953).

Así, el mapa expuesto (Figura 1) ha sido extraído de una publicación del Ministerio de Educación Nacional del año 1953, titulada Nuevas excavaciones arqueológicas en las Canarias Occidentales, que contiene las excavaciones efectuadas en La Gomera, El Hierro, La Palma y Tenerife a partir de 1947, periodo en el que el arqueólogo ejercía su cargo como Comisario Local de la Comisaría General de Excavaciones Arqueológicas.

La realidad esbozada en su prólogo determina el carácter netamente científico del escrito, representando el primer trabajo verdaderamente arqueológico llevado a cabo en las islas: 
No cabían dentro del moderno concepto de esta ciencia los trabajos aislados, realizados sin método, y con una sola preocupación por el objeto. El yacimiento, como tal, no aparecía en ningún intento de estudio arqueológico, y la excavación no se empleó nunca, porque no se puede llamar excavación a la remoción de un yacimiento y menos cuando no se hace seguir de una descripción clara, minuciosa y objetiva. Todo ello produjo lastimosas consecuencias (DIEGO, 1953: 13).

Esta catalogación embrionaria demuestra el papel preponderante que representa las Cañadas del Teide definida como «la más vasta estación arqueológica de Tenerife y acaso lo más alta de Europa»(DiEGO, 1953: 15). A pesar de lo arcaico de la catalogación, es la primera vez que existe una intención oficial por conocer y localizar los entornos arqueológicos de la isla, así como el registro de los materiales obtenidos, refiriendo con minuciosidad cada uno de los yacimientos descritos y localizados, llevando a cabo también su registro material y documentación gráfica.

\section{INVENTARIOS EN EL PERIODO AUTONÓMICO}

Tras la creación del Departamento de Arqueología y Prehistoria de la Universidad de La Laguna a finales de la década de los setenta, se produce el despegue definitivo liderado por las nuevas generaciones de arqueólogas y arqueólogos isleños, bajo la dirección de Manuel Pellicer Catalán y Pilar Acosta Martínez. A su amparo, se llevó a cabo la confección de la Carta Arqueológica del Archipiélago, en vistas a generar nuevas vías de investigación y nuevos ámbitos de actuación (NAVARRO, 1997).

Años más tarde, en 1980, varios miembros del Departamento de Arqueología de la Universidad de La Laguna, María de la Cruz Jiménez, Antonio Tejera y Manuel Lorenzo, elaboraron la Carta Arqueológica de Tenerife, con el objeto de catalogar, fijar y presentar de forma descriptiva el conjunto de yacimientos y materiales respectivos existentes en la isla (JIMÉnEz et al., 1980: 5). Para su confección, se dividió la isla en cuatro zonas, encomendándose a cada uno de ellos un sector concreto: la zona suroeste fue estudiada por Manuel Lorenzo; mientras de las zonas sureste, noreste y noroeste se encargaron $\mathrm{M}^{\mathrm{a}}$ de la Cruz Jiménez y Antonio Tejera (JimÉNEZ et al., 1980: 7).

El trabajo partió de la recopilación de referencias bibliográficas sobre yacimientos; la catalogación de materiales conservados en el Museo Arqueológico de Santa Cruz y Puerto de la Cruz, además de piezas de colecciones privadas; y, finalmente, la visita y estudio in situ de aquellos yacimientos que se pudieron localizar, a los que se sumarían otros desconocidos (JimÉNEZ et al., 1980: 6). Se establecería un orden de prioridades que jerarquizaba los entornos a catalogar, y el hecho más relevante es que presentaba graves problemas de fondo, fundamentalmente por la existencia de errores en las coordenadas UTM, principalmente en los municipios del sur de la isla.

Este ingente análisis debía contar con presupuestos de partida negativos ante las dificultades insoslayables de la arqueología de Tenerife como eran la escasez de estudios estratigráficos, la carencia de una cronología absoluta o relativa, el saqueo, la dificultosa topografía de la isla o una acusada escasez de 
bibliografía reseñable. El catálogo se limita a la denominación de los yacimientos, con una somera descripción en algunos casos y un poco más extendida en otros, sin un criterio claro. Pese a ello, las pesquisas darían como resultado las cartas arqueológicas de varios municipios de la isla (Adeje, Arona, San Miguel, Granadilla, Arico, Fasnia, Güímar, Candelaria, Santa Cruz, El Rosario, Tegueste, Los Silos, La Orotava y La Laguna) (JiMÉnEZ et al., 1980).

Tras esta campaña, la Dirección General de Patrimonio del Gobierno de Canarias solicita al Centro Internacional para la Conservación del Patrimonio (CICOP) ya en el nuevo milenio, la revisión, actualización y elaboración de las cartas arqueológicas y etnográficas de Tenerife, trabajo que continuó presentando las mismas lagunas informativas y errores de coordenadas.

En el mismo orden de ideas, el Museo Arqueológico de Tenerife ha tenido un papel preponderante en este proceso, acometiendo labores de prospección e inventariado a través de la elaboración de la Carta Arqueológica de Arafo y Guía de Isora, así como la del Parque Rural de Teno, a petición del Área de Medio Ambiente del Cabildo.

La siguiente tabla (Tabla 1) recoge la relación de inventarios llevados a cabo en la isla, especificando la fecha de realización, el promotor y el ejecutor del proyecto.

TABLA 3

Relación de los proyectos efectuados en la isla de Tenerife, clasificados por fecha, promotor y ejecutor

\begin{tabular}{|c|c|c|c|}
\hline Proyecto & Fecha & Promotor & Ejecutor \\
\hline $\begin{array}{l}\text { Inventario arqueológico del } \\
\text { Municipio de Guía de Isora }\end{array}$ & 1994 & $\begin{array}{c}\text { Museo } \\
\text { Arqueológico }\end{array}$ & \\
\hline $\begin{array}{l}\text { Inventario arqueológico y } \\
\text { etnográfico de Masca (zona } \\
\text { comprendida entre el Bco. de las } \\
\text { Aneas, carretera de Masca y Bco. del } \\
\text { Natero) }\end{array}$ & 1995 & $\begin{array}{c}\text { Museo } \\
\text { Arqueológico }\end{array}$ & $\begin{array}{c}\text { Museo } \\
\text { Arqueológico }\end{array}$ \\
\hline $\begin{array}{l}\text { Prospección de Nueva carretera } \\
\text { variante de Tejina TF. } 121 \text { de La } \\
\text { Laguna a Punta del Hidalgo. PK. } \\
7,500 \text { al 9,000. }\end{array}$ & 2000 & $\begin{array}{l}\text { Cabildo Insular } \\
\text { de Tenerife }\end{array}$ & \\
\hline $\begin{array}{l}\text { Inventario de la Comarca de } \\
\text { Acentejo (municipios de Tacoronte, } \\
\text { El Sauzal, la Matanza de Acentejo, } \\
\text { La Victoria de Acentejo, Santa } \\
\text { Úrsula). }\end{array}$ & 20022003 & $\begin{array}{l}\text { Cabildo Insular } \\
\text { de Tenerife }\end{array}$ & CICOP \\
\hline $\begin{array}{l}\text { Inventario arqueológico de la } \\
\text { margen izquierda del Bco. de Agua } \\
\text { de Dios (Tejina, La Laguna) }\end{array}$ & 2003 & $\begin{array}{l}\text { Cabildo Insular } \\
\text { de Tenerife }\end{array}$ & $\begin{array}{c}\text { Profesionales } \\
\text { liberales }\end{array}$ \\
\hline
\end{tabular}


Gisela de la Guardia Montesdeoca

\begin{tabular}{|c|c|c|c|}
\hline Proyecto & Fecha & Promotor & Ejecutor \\
\hline $\begin{array}{l}\text { Inventario de la franja costera de } \\
\text { la isla de Tenerife (municipios de } \\
\text { Santa Cruz de Tenerife, El Rosario, } \\
\text { Candelaria, Arafo, Güímar, Fasnia } \\
\text { y Arico, entre la línea litoral y } \\
\text { una línea imaginaria paralela a la } \\
\text { Autopista TF } 1 \text { que discurre a } 100 \mathrm{~m} \\
\text { por encima de la misma) }\end{array}$ & 20032004 & $\begin{array}{l}\text { Cabildo Insular } \\
\text { de Tenerife }\end{array}$ & $\begin{array}{l}\text { Museo } \\
\text { Arqueológico de } \\
\text { Tenerife }\end{array}$ \\
\hline $\begin{array}{l}\text { Inventario del Valle de La Orotava } \\
\text { (municipios de La Orotava, } \\
\text { Puerto de la Cruz y Los Realejos } \\
\text { (exceptuando la zona de Tigaiga } \\
\text { e Icod el Alto, es decir, la parte del } \\
\text { municipio fuera del Valle) }\end{array}$ & 2004 & $\begin{array}{l}\text { Cabildo Insular } \\
\text { de Tenerife }\end{array}$ & CICOP \\
\hline $\begin{array}{l}\text { Inventario Arqueológico del } \\
\text { Territorio limitado por la Autopista } \\
\text { del Sur, carretera C } 822 \text { y carretera } \\
820 \text { de los municipios del Sur y } \\
\text { Oeste de Tenerife, } 2^{\mathrm{a}} \text { Fase, Términos } \\
\text { Municipales de Granadilla de } \\
\text { Abona, San Miguel de Abona y } \\
\text { Arona. }\end{array}$ & 20042005 & $\begin{array}{l}\text { Cabildo Insular } \\
\text { de Tenerife }\end{array}$ & $\begin{array}{l}\text { Museo } \\
\text { Arqueológico de } \\
\text { Tenerife }\end{array}$ \\
\hline $\begin{array}{l}\text { Inventario del Valle de Güímar } \\
\text { (municipios de Candelaria, Arafo y } \\
\text { Güímar, exceptuando la comarca de } \\
\text { Agache) }\end{array}$ & 20052006 & $\begin{array}{l}\text { Cabildo Insular } \\
\text { de Tenerife }\end{array}$ & $\begin{array}{l}\text { Museo } \\
\text { Arqueológico de } \\
\text { Tenerife }\end{array}$ \\
\hline $\begin{array}{l}\text { Inventario Arqueológico del } \\
\text { Territorio limitado por la Autopista } \\
\text { del Sur, carretera C } 822 \text { y carretera } \\
820 \text { de los municipios del Sur y Oeste } \\
\text { de Tenerife, III }{ }^{\mathrm{a}} \text { Fase, Términos } \\
\text { Municipales de Adeje, Guía de Isora } \\
\text { Santiago del Teide. }\end{array}$ & 20052006 & $\begin{array}{l}\text { Cabildo Insular } \\
\text { de Tenerife }\end{array}$ & $\begin{array}{l}\text { Museo } \\
\text { Arqueológico de } \\
\text { Tenerife }\end{array}$ \\
\hline $\begin{array}{l}\text { Inventario de zona afectada por el } \\
\text { incendio de } 2007 \text { (zona alta de los } \\
\text { TT.MM. de El Tanque, Garachico, } \\
\text { San Juan de la Rambla, Icod de los } \\
\text { Vinos, Los Realejos, Santiago del } \\
\text { Teide) }\end{array}$ & 20072008 & $\begin{array}{l}\text { Cabildo Insular } \\
\text { de Tenerife }\end{array}$ & $\begin{array}{l}\text { Profesionales } \\
\text { liberales }\end{array}$ \\
\hline $\begin{array}{l}\text { Inventario de la Isla Baja (TT.MM. } \\
\text { de El Tanque, Garachico, Buenavista } \\
\text { del Norte, Los Silos e Icod de los } \\
\text { Vinos) }\end{array}$ & 20072008 & $\begin{array}{l}\text { Cabildo Insular } \\
\text { de Tenerife }\end{array}$ & $\begin{array}{l}\text { Profesionales } \\
\text { liberales }\end{array}$ \\
\hline $\begin{array}{l}\text { Inventario de Tegueste y } \\
\text { Cristóbal de La Laguna }\end{array}$ & 20072008 & $\begin{array}{l}\text { Cabildo Insular } \\
\text { de Tenerife }\end{array}$ & $\begin{array}{l}\text { Profesionales } \\
\text { liberales }\end{array}$ \\
\hline $\begin{array}{l}\text { Inventario arqueológico del Parque } \\
\text { Rural de Teno }\end{array}$ & 20072008 & $\begin{array}{l}\text { Cabildo Insular } \\
\text { de Tenerife }\end{array}$ & $\begin{array}{c}\text { Profesionales } \\
\text { liberales }\end{array}$ \\
\hline $\begin{array}{l}\text { Inventario arqueológico de } \\
\text { Granadilla de Abona. }\end{array}$ & 2008 & $\begin{array}{l}\text { Ayuntamiento } \\
\text { de Granadilla de } \\
\text { Abona }\end{array}$ & $\begin{array}{l}\text { Universidad de } \\
\text { La Laguna }\end{array}$ \\
\hline
\end{tabular}


Proceso de inventario y catalogación del pasado arqueológico en la isla de Tenerife

\begin{tabular}{|c|c|c|c|}
\hline Proyecto & Fecha & Promotor & Ejecutor \\
\hline $\begin{array}{l}\text { Evaluación y diagnóstico del } \\
\text { patrimonio cultural del sur de la } \\
\text { isla de Tenerife (municipios de } \\
\text { Güímar (sólo Agache), Fasnia, } \\
\text { Arico, Adeje y Vilaflor): para los } \\
3 \text { primeros municipios, el ámbito } \\
\text { estaba comprendido entre la costa } \\
\text { y la carretera general del sur; para } \\
\text { Adeje y Vilaflor, entre la costa y el } \\
\text { límite del Parque Natural de Corona } \\
\text { Forestal y la Reserva Natural } \\
\text { Especial del Bco. del Infierno) }\end{array}$ & 20082009 & $\begin{array}{l}\text { Cabildo Insular } \\
\text { de Tenerife }\end{array}$ & $\begin{array}{l}\text { Profesionales } \\
\text { liberales }\end{array}$ \\
\hline $\begin{array}{l}\text { Inventario patrimonial de la } \\
\text { comarca de Agache y los municipios } \\
\text { de Fasnia y Arico (entre la carretera } \\
\text { general del sur y el límite del Parque } \\
\text { Natural de Corona Forestal) }\end{array}$ & 2014 & $\begin{array}{l}\text { Cabildo Insular } \\
\text { de Tenerife }\end{array}$ & $\begin{array}{l}\text { Profesionales } \\
\text { liberales }\end{array}$ \\
\hline $\begin{array}{l}\text { Inventario patrimonial de Santiago } \\
\text { del Teide }\end{array}$ & 2015 & $\begin{array}{l}\text { Cabildo Insular } \\
\text { de Tenerife }\end{array}$ & $\begin{array}{l}\text { Profesionales } \\
\text { liberales }\end{array}$ \\
\hline $\begin{array}{l}\text { Inventario proyecto de Playa de } \\
\text { Santo Domingo (La Guancha) }\end{array}$ & 2015 & $\begin{array}{l}\text { Proyectos } \\
\text { ejecución }\end{array}$ & Profesional liberal \\
\hline $\begin{array}{l}\text { Inventario patrimonial de comarca } \\
\text { de Acentejo (términos municipales } \\
\text { de Tacoronte, El Sauzal, La Matanza } \\
\text { de Acentejo, La Victoria de Acentejo } \\
\text { y Santa Ursula, entre el límite del } \\
\text { Paisaje Protegido Costa de Acentejo } \\
\text { y el Paisaje Protegido de Las } \\
\text { Lagunetas) }\end{array}$ & 20162017 & $\begin{array}{l}\text { Cabildo Insular } \\
\text { de Tenerife }\end{array}$ & $\begin{array}{l}\text { Profesionales } \\
\text { liberales }\end{array}$ \\
\hline $\begin{array}{l}\text { Inventario arqueológico del } \\
\text { Complejo medioambiental de Arico }\end{array}$ & Sin fecha & $\begin{array}{c}\text { Área de Medio } \\
\text { Ambiente del } \\
\text { Cabildo Insular } \\
\text { de Tenerife }\end{array}$ & $\begin{array}{l}\text { Profesionales } \\
\text { liberales }\end{array}$ \\
\hline $\begin{array}{l}\text { Inventario del Paisaje Protegido } \\
\text { Costa de Acentejo }\end{array}$ & 2017 & $\begin{array}{l}\text { Área de Medio } \\
\text { Ambiente del } \\
\text { Cabildo Insular } \\
\text { de Tenerife }\end{array}$ & $\begin{array}{l}\text { Profesionales } \\
\text { liberales }\end{array}$ \\
\hline Inventario del Cabildo de Tenerife & 2018 & $\begin{array}{l}\text { Cabildo de } \\
\text { Tenerife }\end{array}$ & $\begin{array}{l}\text { Profesionales } \\
\text { liberales }\end{array}$ \\
\hline
\end{tabular}

Fuente: Cabildo Insular de Tenerife.

Con la finalidad de minimizar los riesgos que se cernían sobre el precario patrimonio cultural de la isla de Tenerife, en el año 2003 se inició un programa sistemático de prospecciones en el conjunto de la isla, dando como resultado una «herramienta fundamental para abordar la gestión cotidiana del Patrimonio Histórico de Tenerife» (CABRERA y SOLER, 2018: 55).

A finales del año 2017, el Cabildo de Tenerife presentó el resultado de su labor de inventariado de recursos y bienes patrimoniales, con un total de 27836 
referencias, de las cuales 5437 se corresponden con bienes arqueológicos, 17674 englobadas dentro de la categoría de patrimonio etnográfico y 4725 de patrimonio arquitectónico («Un inventario recoge más de 27.000 recursos patrimoniales de Tenerife», 2017), un titánico proyecto de documentación acometido por un arqueólogo independiente, que efectuó el más que necesario análisis de la información generada en las prospecciones sistemáticas llevadas a cabo en la isla y que confería en última instancia, una realidad profundamente fragmentaria.

El planteamiento seguido obedece a un intento de transferencia de información sobre patrimonio histórico del Cabildo Insular de Tenerife a los municipios, siguiendo cuatro líneas de actuación principales:

- Organización de la información sobre patrimonio cultural (patrimonio arqueológico, etnográfico y arquitectónico).

- Trasferencia de información relevante:

- Fichas de registro de los bienes patrimoniales a disposición de los ayuntamientos.

- $\quad$ Archivo KMZ para la visualización de los datos georreferenciados.

- Cartografía temática.

- Asistencia técnica a los técnicos municipales.

- Propuesta de servicios mediante convenio:

- Prevención.

- Celebración de jornadas y talleres.

- Contratación de arqueólogos, sin coste para la administración municipal.

- Plazos más cortos para la realización de informes.

- Flujo continuo de información.

- Seguridad jurídica.

\section{CONCLUSIONES}

El presente trabajo desgrana la evolución del proceso de catalogación e inventariado en la isla de Tenerife, a partir de los datos recogidos en la investigación especializada, así como las fuentes consultadas en el Archivo de la Dirección General de Patrimonio Cultural. A partir del análisis de las fuentes planteadas se puede establecer la conclusión por la cual las intervenciones arqueológicas del último lustro en la isla de Tenerife confirman la tendencia ya indicada por Pérez (2014), con un predominio de las labores de prospección, al amparo de la preponderancia otorgada al proceso de inventariado, así como las distintas labores insertas en lo que conocemos como arqueología preventiva.

Desde otra perspectiva y siempre partiendo de los datos del archivo de la Dirección General de Patrimonio Cultural, evidenciamos ausencias en la entrega de algunas memorias de intervención, siendo esta una acción preceptiva. Este documento, que es un valioso receptáculo de conocimiento, debería ser, no sólo entregado como estipula la ley, sino difundido a la ciudadanía en plataformas o redes específicas mediante su publicación, labor que en los años 80 y 90 del pasado siglo xx desempeñaba la colección Investigaciones Arqueológicas en Canarias.

La política llevada a cabo en la isla de Tenerife, en la que prima la labor de inventariado y catalogación, es necesaria e indispensable para establecer una hoja de ruta futura y, sobre todo, para asegurar la preservación del patrimonio 
arqueológico. La contrapartida a esta ingente cantidad de información y de esfuerzos requeridos es que todo ese conocimiento, confidencial por otra parte (CABRERA, 2010), permanece ajeno completamente a la ciudadanía. Todo ello debe entenderse en una isla que por sus especiales características patrimoniales no ha permitido o no se ha permitido la difusión de su patrimonio arqueológico, a diferencia de otras islas (CABRERA, 2010). No puede obviarse el hecho de que es una cuestión prioritaria retomar proyectos investigadores de amplio espectro como base de un proceso de gestión del patrimonio que debe basarse en la generación de estrategias planificadas, consensuadas y participativas. Al respecto, de nuevo remitiéndonos al trabajo de PÉREz (2014: 257), se hace necesario la elaboración de un registro de actividades llevadas a cabo en las islas, que reúna la información actualmente dispersa en torno a las intervenciones arqueológicas realizadas, con el fin de unificar fuentes, y desde nuestro punto de vista, que pueda ser visible y justifique de alguna manera las acciones realizadas en suelo canario, obedeciendo al criterio de transparencia que debe regir el funcionamiento público.

\section{REFERENCIAS LEGISLATIVAS}

Real Cédula de 6 de julio de 1803, por la cual se aprueba y manda observar la Instrucción formada por la Real Academia de la Historia sobre el modo de recoger y conservar los monumentos antiguos descubiertos o que se descubran en el Reino (1803).

Ley de 7 de julio de 1911, que estableció las reglas a que han de someterse las excavaciones artísticas y científicas y la conservación de las ruinas y antigüedades (1911). Boletín de la Real Academia de la Historia.

Recomendación que define los Principios Internacionales que deberían aplicarse a las Excavaciones Arqueológicas. Nueva Delhi. 5 de diciembre de 1956.

Convenio Europeo sobre la protección del Patrimonio Arqueológico. La Valetta. 16 de enero de 1992

Decreto 31/1993, de 5 de marzo, por el que se establece el régimen general de ayudas y subvenciones de la Administración Autonómica de Canarias (1993). Boletín Oficial de Canarias.

Ley 4/1999, de 15 de marzo, de Patrimonio Histórico de Canarias (1999). Boletín Oficial del Estado.

Decreto 262/2003, de 23 de septiembre, por el que se aprueba el Reglamento sobre intervenciones arqueológicas en la Comunidad Autónoma de Canarias (2003). Boletín Oficial de Canarias.

Ley 11/2019, de 25 de abril, de Patrimonio Cultural de Canarias (2019). Boletín Oficial del Estado.

\section{REFERENCIAS}

BANKs, M. (2010): Los datos visuales en investigación cualitativa, Morata, Madrid.

Bugella Altamirano, M. (2017): «La protección jurídica del Patrimonio Arqueológico. De la Real Cédula de 1803 a la Guerra Civil», Patrimonio 
Cultural y Derecho, 21: 319-334.

CABrera, J.C. (2010): «La compleja puesta en valor del patrimonio arqueológico. El caso de Tenerife», Canarias Arqueológica: Arqueología-Bioantropología, 18: 199214.

Cabrera, J.C.; Soler Segura, J. (2018): «El inventario insular de bienes patrimoniales», Revista BIC, 1: 51-61.

Cabrera, J.C. (2019): «Servicio de Patrimonio del Cabildo, base de la gestión y protección de los bienes insulares», Revista BIC, 2: 55-63.

Diego Cuscoy, L. (1953): Nuevas excavaciones arqueológicas en las Canarias Occidentales: yacimientos de Tenerife y La Gomera (1947-1951), Ministerio de Educación Nacional, Comisaría General de Excavaciones Arqueológicas, Madrid.

Jiménez Díez, J.A.; Mederos Martín, A. (coord.) (2001): Comisión de antigüedades de la Real Academia de la Historia. Baleares, Canarias, Ceuta y Melilla. Extranjero. Catálogo e índices. Real Academia de la Historia, Madrid.

JiméneZ, M.; TejerA, A.M.; Lorenzo, M. (1980): Carta arqueológica de Tenerife, Aula de Cultura de Tenerife, Santa Cruz de Tenerife.

JiMÉNEZ GoNZÁLEZ, J.J. (1995): «Una perspectiva histórica analítica del patrimonio arqueológico y etnoarqueológico canario», Eres. Arqueología/Bioantropología, 5: 23-31.

Martín Ruiz, J.M.; SÁnchez Bandera, P.J.; Martín Ruiz, J.A. (1995): «La carta arqueológica como instrumento de investigación y gestión patrimonial. El caso del Valle de Abdalajís», Mainake, 17-18: 243-260.

NAvArro, J.F. (1997): «Arqueología de las Islas Canarias», Espacio, Tiempo y Forma, Serie I, Prehistoria y Arqueología, 10: 447-478.

Navarro, J.F.; Tejera, A.; Cuenca Sanabria, J. (2005): «Veinte años después: la gestión del patrimonio arqueológico canario desde las transferencias a la comunidad autónoma», en V Jornadas de Patrimonio Histórico: la arqueología canaria. Análisis de partida, Cabildo de Lanzarote, Arrecife.

PÉREZ, E. (2014): Diagnosis y estrategias para una gestión del patrimonio arqueológico de las Islas Canarias, Tesis doctoral inédita, Universidad de La Laguna.

Querol Fernández, M.A. (1992): «Los mecanismos de protección del patrimonio arqueológico», Trabajos de Prehistoria, 49: 27-34.

Querol FernÁNDEZ, M.A. (1995): «Intervenciones y destrucciones: la gestión de la actividad arqueológica», Boletín de la ANABAD, 45 (3): 131-168.

Querol Fernández, M.A. (2010): Manual de gestión del patrimonio cultural, Akal, Madrid.

Ramos Millán, A.; Tapia Espinosa, A.; Aznar Pérez, J.C.; Osuna Vargas, M.M. (1998): «El impacto arqueológico desde perspectivas conservacionistas. La autovía del Mediterráneo, Baza - Puerto Lumbreras. Tramo Puerto Lumbreras (Murcia)», en M. Lechuga Galindo y M.B. SÁnchez GonzÁlez (eds.), Cuartas Jornadas de Arqueología Regional, Gobierno de la Región de Murcia, Murcia: 512-526.

Rodríguez de GuzMán SÁnchez, S. (2004): «El planeamiento como modo de protección del Patrimonio Arqueológico: el caso de la Comunidad Autónoma de Andalucía», en Actas de las Primeras Jornadas de Patrimonio Arqueológico de la Comunidad de Madrid, Comunidad de Madrid, Madrid: 15-38.

TEjerA, A. (1992): «La investigación arqueológica en las Canarias, 1992», Almogaren, 23: $77-84$. 
